\title{
Emodin protects H9c2 cells from hypoxia-induced injury by up-regulating miR-138 expression
}

\author{
Xuezhi Zhang ${ }^{1}$, Qiaoji Qin ${ }^{1}$, Hongyan Dai ${ }^{2}$, Shanglang Cai ${ }^{3}$, Changyong Zhou ${ }^{1}$ and Jun Guan ${ }^{2}$ \\ ${ }^{1}$ Department of Emergency Internal Medicine, The Affiliated Hospital of Qingdao University, Qingdao, China \\ ${ }^{2}$ Department of Cardiology, Qingdao Municipal Hospital, Qingdao, China \\ ${ }^{3}$ Department of Cardiology, The Affiliated Hospital of Qingdao University, Qingdao, China
}

\begin{abstract}
Myocardial infarction (MI) is a common presentation for ischemic heart disease, which is a leading cause of death. Emodin is a Chinese herbal anthraquinone used in several diseases. However, the effect of emodin in hypoxia-induced injury in cardiomyocytes has not been clearly elucidated. Our study aimed to clarify the functions of emodin in hypoxia-induced injury in rat cardiomyocytes $\mathrm{H} 9 \mathrm{c} 2$ and explore the underlying mechanism. The effects of emodin on cell viability and apoptosis were analyzed by the Cell counting kit- 8 assay and flow cytometry assay, respectively. The cell proliferation- and cell apoptosisrelated proteins were detected by western blot. qRT-PCR was used to determine the relative expression of miR-138. Cell transfection was performed to alter miR-138 and MLK3 expression. miR-138 target was performed by dual luciferase activity assay. Sirt1/AKT and Wnt/ß-catenin pathways-related factors phosphorylation were analyzed by western blot. Emodin inhibited hypoxia-induced injury in $\mathrm{H} 9 \mathrm{c} 2$ cells by promoting cell viability and reducing cell apoptosis. miR-138 was down-regulated by hypoxia treatment but up-regulated by emodin. Up-regulation of miR-138 alleviated hypoxia-induced cell injury. Down-regulation of miR-138 attenuated the growth-promoting effect of emodin on hypoxia-induced injury, whereas up-regulation of miR-138 enhanced the growth-promoting effects of emodin. The underlying mechanism might be by inactivating Sirt1/AKT and Wnt/ $\beta$-catenin pathways. MLK3 was negatively regulated by miR-138 expression and inactivated Sirt $1 / \mathrm{AKT}$ and Wnt/ $\beta$-catenin pathways. Emodin alleviated hypoxia-induced injury in $\mathrm{H} 9 \mathrm{c} 2$ cells via up-regulation of miR-138 modulated by MLK3, as well as by activating Sirt1/AKT and Wnt/ $\beta$-catenin pathways.
\end{abstract}

Key words: Emodin; Myocardial infarction; Hypoxia injury; miR-138; Sirt1/AKT pathway; Wnt/ß-catenin pathway

\section{Introduction}

Myocardial infarction (MI) is a common presentation for ischemic heart disease and coronary artery disease, which is a leading cause of death among all cardiovascular diseases (1). The classification differentiates between type $1 \mathrm{MI}$, due to thrombosis of an atherosclerotic plaque, and type $2 \mathrm{MI}$, due to the imbalanced supply and demand of myocardial blood that might be triggered by many acute medical and surgical conditions (2). The immediate revascularization and optimal medical therapy may improve prognosis in these patients. In spite of advances of medical and interventional treatment, mortality in patients with acute MI remains high (3). Therefore, new medicines and new therapies are urgently needed.

Emodin (1,3,8-trihydroxy-6-methyl-9,10-anthracenedione, $\mathrm{C}_{15} \mathrm{H}_{10} \mathrm{O}_{5}$ ) is a Chinese herbal anthraquinone isolated from some traditional Chinese herbs including Rhubarb and Polygonum cuspidatum. Emodin exhibits numerous biological activities, such as anti-tumor (4), anti-viral (5), anti-bacterial (6), anti-inflammation (7), and anti-oxidation (7).
It was reported that emodin had potential effects to attenuate lipopolysaccharide (LPS) and hypoxia/reoxygenation-induced intestinal epithelial barrier dysfunction (8). Aloe-emodin could suppress hypoxia-induced retinal angiogenesis by inhibition of the hypoxia-inducible factor (HIF-1 $\alpha$ )/vascular endothelial growth factor (VEGF) pathway (9). However, limited studies were performed to investigate the role of emodin in $\mathrm{MI}$ and other cardiovascular diseases.

microRNAs (miRNAs), a class of small noncoding RNAs, can negatively regulate gene expression at the post-transcriptional level (10). miRNAs play a pivotal role in drug efficacy and toxicity and have potential clinical implications for personalized medicine by regulating the expression of pharmacogenomic-related genes (11). A variety of miRNAs were found to modulate the efficacy of emodin, such as miR-126 (12), miR-34a (13), and miR199a (14). Among these identified miRNAs, miR-138 was reported to protect cardiomyocytes against hypoxiainduced injury and considered an important regulator in

Correspondence: Jun Guan: <guanjun514@sina.com> 
cellular signaling pathways (15). Importantly, miR-138 has proven to be crucial in cardiac cell fate decisions including cardiac conduction, regulation of cardiac patterning, management of angiogenesis, and protection against ischemia (16). Whether emodin regulates miR-138 expression and affects hypoxia-induced injury of cardiomyocytes by regulating miR-138 expression have not been well studied. Therefore, our study aimed to explore the effect of emodin on hypoxia-induced injury in $\mathrm{H} 9 \mathrm{c} 2$ cells and in which process the role of miR-138 regulated by emodin was investigated.

Wnt/ $\beta$-catenin and sirtuin 1 (Sirt1)/protein kinase B (AKT) signaling pathway regulated a broad range of cell processes, such as cell proliferation, apoptosis, invasion, and differentiation $(17,18)$. Meanwhile, miR-138 was observed to have close correlation with Sirt1/AKT and Wnt/ $\beta$-catenin signal pathways. For example, miR-138-5p promoted tumor necrosis factor-alpha (TNF- $\alpha$ )-induced apoptosis in human intervertebral disc degeneration by targeting Sirt1 (19). In addition, TUG1-miR-138-5p-Sirt1-Wnt/ $\beta$-catenin signaling pathway axis provided new light on the diagnosis and treatment for cervical cancer patients (20). Therefore, in our study, the potential regulation between miR-138 and these signal pathways was also explored.

\section{Material and Methods}

\section{Cell culture and treatment}

Rat cardiomyocytes cell line $(\mathrm{H} 9 \mathrm{c} 2)$, purchased from the American Type Culture Collection (ATCC, USA), was seeded into flasks $\left(1 \times 10^{4}\right.$ cells $\left./ \mathrm{mL}\right)$ in Dulbecco's modified Eagle medium (DMEM, Gibco, USA) containing 10\% (v/v) fetal bovine serum (FBS, Life Science, USA), $100 \mathrm{U} / \mathrm{mL}$ penicillin, and $100 \mu \mathrm{g} / \mathrm{mL}$ streptomycin and incubated at $37^{\circ} \mathrm{C}$ under humid conditions in an atmosphere of $95 \%$ air and $5 \% \mathrm{CO}_{2}$. $\mathrm{H} 9 \mathrm{c} 2$ cells were incubated in a hypoxic incubator containing $1 \% \mathrm{O}_{2}, 94 \% \mathrm{~N}_{2}$, and $5 \% \mathrm{CO}_{2}$ for inducing hypoxia injury. Firstly, the $\mathrm{H} 9 \mathrm{c} 2$ cells were exposed in a hypoxia environment for $4,8,16,24$, and $48 \mathrm{~h}$ for choosing a useful hypoxia treatment time for the following experiments. At the end of each time interval for hypoxia treatment, the incubator was refilled with $\mathrm{O}_{2}$ to the normal condition for reoxygenation.

Emodin (Sigma-Aldrich, USA (ref: E7881, $\geqslant 90 \%$ (HPLC)) was dissolved in dimethyl sulfoxide (DMSO, Sigma Aldrich) at concentration of $100 \mathrm{mM}$ and was used to treat cells at concentrations ranging from $5 \mu \mathrm{M}$ to $20 \mu \mathrm{M}$.

\section{Cell counting kit-8 (CCK-8) assay}

H9c2 cells were seeded in a 96-well plate with 5000 cells/well. After treatment, $20 \mu \mathrm{L}$ CCK-8 solution (Beyotime, China) was added to the culture medium, and the 96-well plate was incubated for $1 \mathrm{~h}$ at $37^{\circ} \mathrm{C}$ in humidified $95 \%$ air and $5 \% \mathrm{CO}_{2}$. The absorbance was measured at $450 \mathrm{~nm}$ using a Microplate Reader (Bio-Rad, USA).

\section{Apoptosis assay}

The apoptotic cell rate was analyzed by annexin V-fluorescein isothiocyanate (FITC)/propidium iodide (PI) apoptosis detection kit (Beijing Biosea Biotechnology, China) combined with flow cytometry analysis. Cells were rinsed in PBS and then stained in PI and FITC-annexin $V$ solution in the presence of $50 \mu \mathrm{g} / \mathrm{mL}$ RNase A (SigmaAldrich). After incubation for $2 \mathrm{~h}$ at room temperature in the dark, apoptotic cell rate was determined using a FACS can (Beckman Coulter, USA). The data were analyzed using FlowJo software (TreeStar, USA).

\section{miRNA silencing and overexpression}

miR-138 inhibitor, miR-138 mimic, pc-MLK3, and the negative control (NC) were synthesized by GenePharma Co. (China). The sequence of rno miR-138 inhibitor was: 5'-CGGCCUGAUUCACAACACCAGCU-3'; the sequence of rno miR-138 mimic was: 5'-AGCUGGUGUUGUGAAU CAGGCCG-3'; the sequence of rno NC was: $5^{\prime}$-UCAC AACCUCCUAGAAAGAGUAGA-3'. In addition, the concentration of miR-138 inhibitor, miR-138 mimic, and NC was 150,50 , and $50 \mathrm{nM}$, respectively. Cell transfection was conducted using Lipofectamine 3000 reagent (Invitrogen, USA) following the manufacturer's instructions. In brief, $2 \times 10^{4} \mathrm{H} 9 \mathrm{c} 2$ cells in a 24 -well plate were transfected with indicated plasmid DNA, miRNA mimic $50 \mathrm{nM}$ (GenePharma), miRNA inhibitor $150 \mathrm{nM}$ (GenePharma), and NC $50 \mathrm{nM}$. Cells were collected 24-48 h after transfection for assay.

\section{Quantitative real time polymerase chain reaction (qRT-PCR) analysis}

Total RNA was extracted from $\mathrm{H} 9 \mathrm{c} 2$ cells by Trizol reagent (Invitrogen). For miR-138 detection, reversetranscribed complementary DNA was synthesized with the PrimeScript RT reagent Kit (TaKaRa, China), and qRTPCR was performed with SYBR Premix ExTaq (TaKaRa) with the Stratagene Mx3000P real-time PCR system (Agilent Technologies, Inc., USA). The sequence of miR138 primer was: forward 5'-GCCGCAGCTGGTGTTGT GAAT-3', reverse 5'-GCGAGCACAGAATTAATACGAC-3'. The sequence of U6 was: forward 5'-CTCGCTTCGG CAGCACA-3', reverse 5'-GCGAGCACAGAATTAATACG $A C-3^{\prime}$, and the concentration of primers was $0.2 \mu \mathrm{mol} / \mathrm{L}$. The relative expression ratio of $\mathrm{miR}-138$ was calculated by the $2^{-\Delta \Delta \mathrm{CT}}$ method (21). The reaction parameters were incubation at $95^{\circ} \mathrm{C}$ for $10 \mathrm{~min}$, then 40 cycles of $95^{\circ} \mathrm{C}$ for $15 \mathrm{~s}, 60^{\circ} \mathrm{C}$ for $1 \mathrm{~min}$. The threshold cycle (Ct) is defined as the cycle number at which the fluorescence passed a predetermined threshold. For expression analysis, the experiment was designed to use the matched non-hypoxia cell as the control, so the relative quantification of miR-138 in hypoxia-treated cells was calculated using the equation: amount of target $=2^{-\Delta \Delta \mathrm{Ct}}, \Delta \Delta \mathrm{Ct}=(\mathrm{CtmiR}-138-$ CtU6 $)_{\text {hypoxia }}$ - (CtmiR-138-CtU6 $)_{\text {matched non-hypoxia. }}$ For the 
matched non-hypoxia cell control sample, $\Delta \Delta \mathrm{Ct}$ is zero and $2-\Delta \Delta \mathrm{Ct}$ is 1 . Melting curves were generated and $8 \%$ PAGE electrophoresis was performed for each real-time PCR to verify the amplification of only the desired product. The same method of analysis was used for the group treated with hypoxia and emodin. U6 was the internal control for miR-138 and $\beta$-actin was the internal control for mixed lineage kinase 3 (MLK3).

\section{Western blot}

Cells were collected and lysed in RIPA lysis buffer (Beyotime) supplemented with protease inhibitors (Roche, Switzerland). The equivalent amounts of proteins $(20 \mu \mathrm{g})$ were denatured at $100^{\circ} \mathrm{C}$ in loading buffer for $15 \mathrm{~min}$, resolved on $8-12 \%$ sodium dodecyl sulfate-polyacrylamide gel electrophoresis (SDS-PAGE) gels, and transferred to polyvinylidene fluoride (PVDF) membranes by voltage gradient transfer. The blots were blocked overnight with $5 \%$ non-fat milk. The membrane was incubated with primary antibodies and proper secondary antibodies. The used primary antibodies against p53 (ab131442), p21 (ab109199), cyclin D1 (ab134175), caspase-3 (ab4051), cleaved-caspase-3 (ab49822), caspase-9 (ab202068), cleaved-caspase-9 (ab2324), MLK3 (ab51068), Sirt1 (ab110304), AKT (ab185633), p-AKT (ab131443), Wnt3a (ab28472), $\beta$-catenin (ab32572), and $\beta$-actin (ab8227) purchased from Abcam (UK) were used at the dilution of $1: 1000$. $\beta$-actin was used as a loading control. After washing, the binding antibody was visualized using the enhanced chemiluminescence assay kit (Tiangen Biotechnology Corp., China) according to the manufacturer's instructions.

\section{Dual luciferase activity assay}

The $3^{\prime} U T R$ of MLK3 was generated by PCR and the luciferase reporter constructs with the miR-138 $3^{\prime} U T R$ carrying a putative miR-138-binding site into pMiR-report vector were amplified by PCR. Mutagenesis was performed when the seed region was mutated to remove all complementarity to nucleotides of miR-138. The sequence of miR-138 was 5'-GCCGGACUAAGUGUUGUGGUCGA-3', the sequence of wild type of MLK3 was: 5'-GGGGAAAG GGGCUGACCUCAGGUGUCACCAGCACUUUU-3', the sequence of mutant MLK3 was: 5'-GGGGAAAGGGGCU GACCUCAGGUGUGUUUGAAACUUUU-3'. Cells were cotransfected with the reporter construct, control vector, and miR-138 mimic or scramble using Lipofectamine 3000 (Life Technologies, USA). Reporter assays were done using the dual-luciferase assay system (Promega, USA) following the manufacturer's information. In brief, the firefly luciferase reporter assay is initiated by mixing lysate with Luciferase assay reagent II (LAR II). Upon completion of the firefly luciferase assay, the firefly luminescence is quenched and Renilla luminescence is simultaneously activated by adding Stop \& Glo TM Reagent to the sample tube. The value of luciferase activity was measured.
The results are reported as a ratio of firefly luciferase (Fluc) activity to Renilla luciferase (Rluc) activity.

\section{Statistical analysis}

All data are reported as means $\pm S D$ from three to six samples. Data analysis was performed using GraphPad Prism version 6.0 software (GraphPad Software, USA). Student's $t$-test, one-way analysis of variance, and two-way analysis of variance were performed according to the data characteristics. $P$ values $<0.05$ were considered statistically significant.

\section{Results}

\section{Hypoxia induced $\mathrm{H} 9 \mathrm{c2}$ cell injury}

$\mathrm{H} 9 \mathrm{c} 2$ cells were incubated in medium under the hypoxia condition for different time spans $(0,4,8,16,24$, and $48 \mathrm{~h})$ and then cell viability was determined. The data showed that cell viability was inhibited by hypoxia treatment and the injury was enhanced with extending treatment time $(P<0.01$ or $P<0.001$, Figure $1 A)$. We found that when the hypoxia treatment time was $\geqslant 16 \mathrm{~h}$, cell viability was severely decreased. Therefore, in the following experiment, hypoxia treatment time was set as $16 \mathrm{~h}$. The protein accumulated level showed that p53 rose by 2 times and p21 accumulated level rose by 1.9 times $(P<0.001)$, and cyclin $D 1$ reduced by 0.67 times $(P<0.01$, Figure $1 B)$. Additionally, hypoxia treatment induced cell apoptosis $(P<0.001$, Figure $1 \mathrm{C}$ ) and increased the ratio of cleaved-/ pro-caspases- 3 by 6.1 times and the ratio of cleaved-/ pro-caspases- 9 by 6.4 times detected by western blot $(P<0.001$, Figure 1D). The data suggested that hypoxia induced injury in $\mathrm{H} 9 \mathrm{c} 2$ cells.

\section{Emodin inhibited hypoxia injury in $\mathrm{H} 9 \mathrm{c} 2$ cells}

Emodin exhibited no negative effect on viability of $\mathrm{H} 9 \mathrm{c} 2$ cells at concentrations of 5,10 , and $15 \mu \mathrm{M}$, but cell viability was decreased by 0.82 times by emodin at the concentration of $20 \mu \mathrm{M}$ compared with control (Figure $2 \mathrm{~A}$ ), indicating that the safe concentrations for the study should be less than $20 \mu \mathrm{M}$. The viability of hypoxia-stimulated $\mathrm{H} 9 \mathrm{c} 2$ cells was increased by emodin treatment in a dosedependent manner (Figure 2B), especially by emodin at the concentration of $15 \mu \mathrm{M}$. Therefore, emodin at the concentration of $15 \mu \mathrm{M}$ was used for following experiments. After emodin treatment, the upregulation of p53 and p21, as well as the downregulation of cyclin D1 were repressed $(P<0.001$, Figure $2 C)$. In addition, cell apoptosis was inhibited by emodin $(P<0.01$, Figure 2D). Furthermore, the ratio of cleaved-/pro-caspases-3 was decreased by 0.4 times and the ratio of cleaved-/procaspases -9 decreased by 0.38 times (both $P<0.001$, Figure 2E and F) confirming the result in Figure 2D. These results suggested that hypoxia-induced injuries were inhibited by emodin. 
A

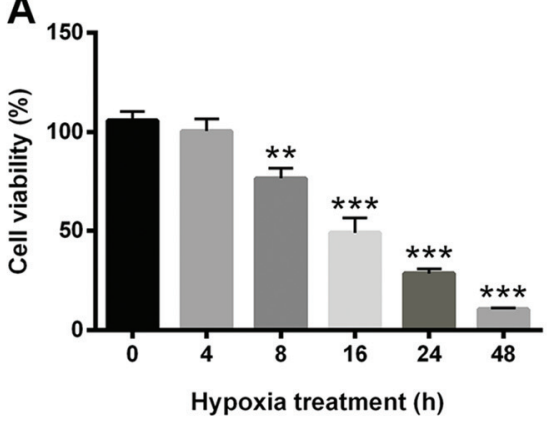

C

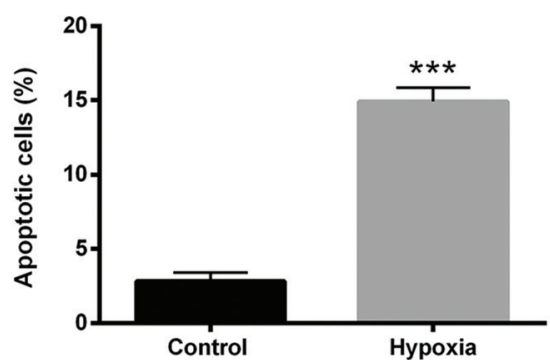

B

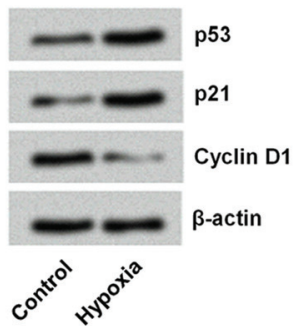

D

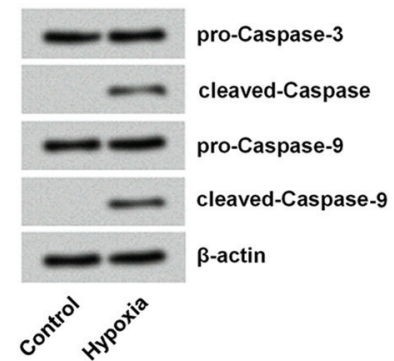

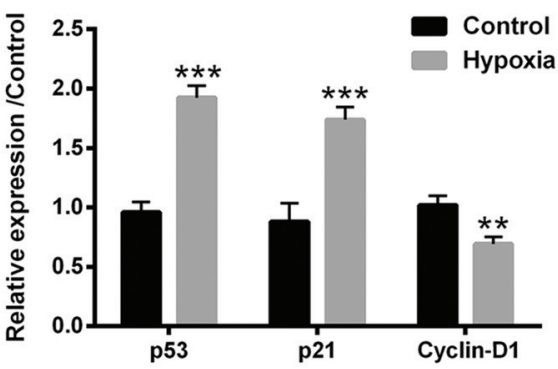

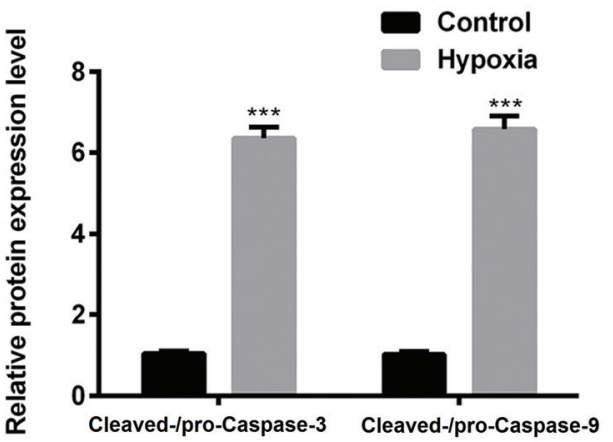

Figure 1. Hypoxia induced injury in $\mathrm{H} 9 \mathrm{c} 2$ cells. $A$, Hypoxia inhibited viability detected by CCK-8 assay. $B$, Hypoxia (16 h) increased antiproliferation protein expression and decreased pro-proliferation protein expression examined by western blot. $C$, Hypoxia (16 h) increased apoptotic cell rate measured by flow cytometry. $D$. Hypoxia $(16 \mathrm{~h})$ activated pro-apoptosis protein expression detected by western blot. Data are reported as means $\pm S D$ of triplicates. Each experiment was performed three times. ${ }^{* *} \mathrm{P}<0.01,{ }^{* * *} \mathrm{P}<0.001$ compared to control (Student's $t$-test and one-way analysis of variance).

\section{Emodin up-regulated miR-138 expression}

The possible mechanism of action and the genetic molecule involved in cytoprotection of emodin were explored. miR-138 was chosen to be investigated according to its close correlation with cardiac cell fate (16). Our result in Figure 3 revealed that miR-138 expression was down-regulated 0.7 times in $\mathrm{H} 9 \mathrm{c} 2$ cells by hypoxia treatment but further up-regulated 2.4 times by emodin administration. The data implied that miR-138 might be involved in the hypoxia-induced injury of cells and regulate the effect of emodin on hypoxia-induced injury.

\section{miR-138 overexpression alleviated hypoxia-induced cell injury}

In order to clarify the functions of miR-138 in hypoxiastimulated $\mathrm{H} 9 \mathrm{c} 2$ cells, miR-138 transfections were carried out. miR-138 expression level was altered by transfection with its corresponding mimic and inhibitor. The relative level of miR-138 was significantly reduced 0.1 times and increased 6.5 times after transfection with miR-138 inhibitor and mimic, respectively $(P<0.05, P<0.001$, Figure $4 A)$. Further experiments were performed and showed that miR-138 overexpression increased cell viability $(P<0.05$, Figure 4B) and decreased cell apoptosis $(P<0.01$,
Figure 4E) in hypoxia-treated cells compared with NC. Meanwhile, the proliferation-related proteins accumulating level shown in Figure 4C and D revealed that miR-138 overexpression significantly up-regulated p53 and p21 accumulation and downregulated cyclin D1 accumulation, indicating that miR-138 had a positive influence on cell proliferation in hypoxia-treated cells. Similarly, western blot results showed that apoptosis-related proteins, cleavedcaspase 3 , and cleaved-caspase-9 were both downregulated by miR-138 overexpression compared with NC in hypoxia-treated cells (Figure 4F-G). On the other hand, miR-138 downregulation led to the opposite results. Taken together, miR-138 overexpression alleviated hypoxiatreated H9c2 cell injury.

\section{Emodin improved hypoxia injury in $\mathrm{H} 9 \mathrm{c} 2$ cells by up-regulating $\mathrm{miR}-138$}

Knockdown of miR-138 induced a decrease in cell viability although cells were treated with emodin in hypoxiastimulated cells $(P<0.01)$, but miR-138 overexpression further enhanced the viability-promoting effect of emodin $(P<0.05$, Figure $5 A)$. Knockdown of miR-138 increased synthesis of p53 2.1 times and p21 by 2.5 times but decreased synthesis of cyclin D1 0.44 times, whereas 

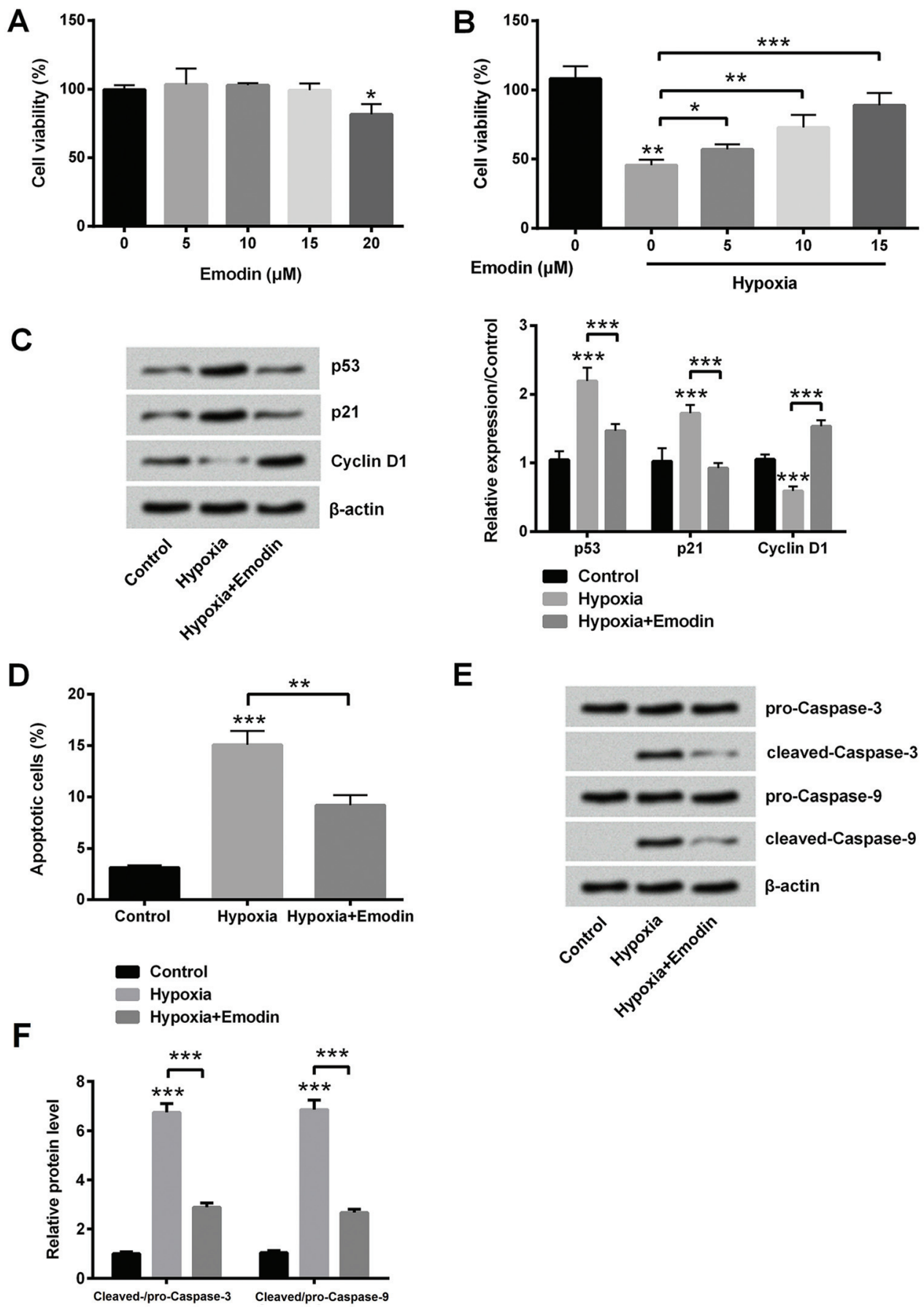

Figure 2. The appropriate concentration of emodin reduced the damaging effect of oxygen-deprivation on H9c2 cells. A, Emodin with concentration less than $20 \mu \mathrm{M}$ had no inhibitory effect on viability of H9c2 cells detected by CCK-8 assay. B, Emodin promoted viability of oxygen-deprived $\mathrm{H} 9 \mathrm{c} 2$ cells in a dose-dependent manner detected by CCK-8 assay. Emodin treatment $(C)$ increased the pro-proliferation protein expression and decreased the anti-proliferation protein, $(D)$ declined apoptotic cell rate, and $(E-F)$ inhibited the pro-apoptosis protein expression. The expression of protein level was detected by western blot and cell apoptosis was measured by flow cytometry. Data are reported as means $\pm S D$ of triplicates. Each experiment was performed three times. ${ }^{*} P<0.05,{ }^{*} P<0.01$, ${ }^{\star * *} \mathrm{P}<0.001$ compared to control or as indicated (one-way analysis of variance). 

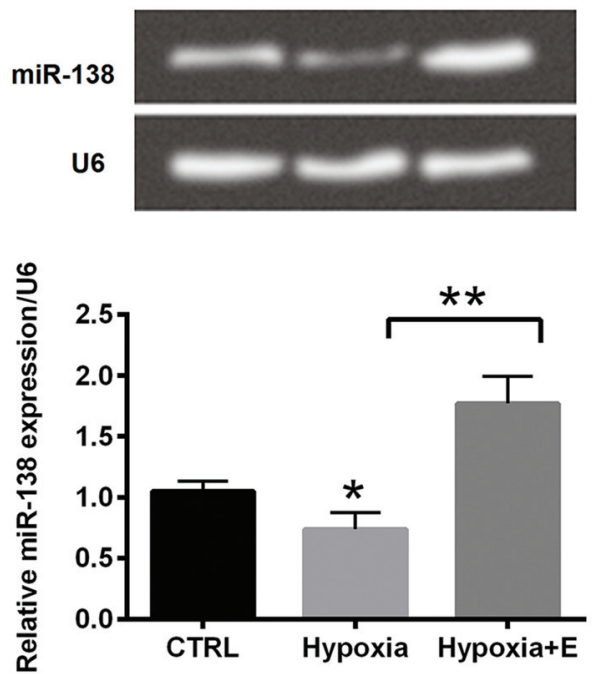

Figure 3. Relative expression of miR-138 was inhibited by hypoxia treatment but then increased by emodin $(E)$ treatment detected by quantitative real-time polymerase chain reaction. Data are reported as means $\pm S D$ of triplicates. Each experiment was performed three times. ${ }^{*} P<0.05$, ${ }^{* *} P<0.001$ compared to control or as indicated (one-way analysis of variance).

up-regulating miR-138 expression exhibited the contrary effects (Figure 5B and C). miR-138 silence impaired the apoptosis-inhibitory effect of emodin while miR-138 overexpression promoted the apoptosis-inhibitory effect of emodin on hypoxia-induced cell injury $(P<0.05$, Figure 5D). Furthermore, the accumulated level of cleaved-caspase- 3 and cleaved-casapase- 9 was upregulated by miR-138 inhibitor $(P<0.001)$ and decreased by miR-138 overexpression (both $P<0.05$, Figure $5 E$ and $F$ ). The results indicated that emodin might ameliorate hypoxia injury in $\mathrm{H} 9 \mathrm{c} 2$ cells by up-regulating miR-138 expression.

\section{Emodin activated Sirt1/AKT and Wnt//-catenin signaling pathways by up-regulating miR-138}

The underlying mechanism of miR-138 in the function of emodin was investigated by western blot. Two pathways, Sirt1/AKT and Wnt/ $\beta$-catenin, were found to be related with $\mathrm{MI}(22,23)$. Hypoxia treatment decreased phosphorylation of Sirt1 $(P<0.001)$ and p-AKT $(P<0.05$, Figure $6 \mathrm{~A})$. Besides, hypoxia treatment diminished the accumulated level of Wnt3a $(P<0.01)$ and $\beta$-catenin $(P<0.05$, Figure $6 B)$. However, emodin treatment activated Sirt1/AKT and Wnt/ $\beta$-catenin pathways by increasing accumulated level of Sirt1, p-AKT, Wnt3a, and $\beta$-catenin (all $P<0.001$, Figure $6 A$ and $B$ ). Interestingly, the two pathways were inactivated and promoted in emodin-treated cells when miR-138 was knocked down and overexpressed, respectively (Figure 6). We speculated that emodin activated Sirt1/AKT and Wnt/ $\beta$-catenin signaling pathways possibly by up-regulating miR-138.

\section{Emodin activated Sirt1/AKT and Wnt/ $\beta$-catenin signaling pathways by down-regulating MLK3}

As shown in the luciferase results, MLK3 was proven to be a target of miR-138 (Figure 7A). Further results from Figure $7 \mathrm{~B}$ demonstrated that MLK3 was negatively regulated by miR-138 overexpression $(P<0.001)$. In order to identify the functions of MLK3 in hypoxiatreated $\mathrm{H} 9 \mathrm{c} 2$ cells, pc-MLK3 was transfected. Upregulation of MLK3 by transfection with pc-MLK3 $(P<0.001)$ indicated the high transfection efficiency (Figure 7C). Co-transfection with miR-138 mimic and pc-MLK3 downregulated the accumulated level of Sirt1, p-AKT, Wnt3a, and $\beta$-catenin (Figure 7D and E) compared with transfection with miR-138 mimic and pcDNA3.1 in hypoxia-treated cells. These results demonstrated that overexpression of MLK3 inactivated Sirt1/AKT and Wnt/ $\beta$-catenin signaling pathways, and emodin activated Sirt1/AKT and $\mathrm{Wnt} / \beta$-catenin signaling pathways by down-regulating MLK3.

\section{Discussion}

Traditionally, emodin has been used as an active ingredient for many Chinese herbal laxatives; however, currently, significant development has been made in investigating its biological effects at cellular and molecular levels and it is emerging as an important therapeutic agent in various fields (24). The present study demonstrated that emodin inhibited hypoxia-induced injury in $\mathrm{H} 9 \mathrm{c} 2$ cells via up-regulation of miR-138 as well as activating Sirt1/AKT and $\mathrm{Wnt} / \beta$-catenin pathways. Our study might provide a new perspective of the modulatory effects of emodin on hypoxia-induced injury in MI, which may widen the research scope of emodin and have the important implication in its future clinical use.

$\mathrm{H} 9 \mathrm{c} 2$ cells stimulated with hypoxia exhibited a decreased viability and increased apoptosis, indicating that cell injury was successfully induced. However, emodin treatment increased viability and decreased apoptosis, alleviating the hypoxia-induced injury.

p53, p21, and cyclin D1 are cell cycle-associated proteins $(25,26)$. p53 exerts anti-proliferative effects in response to various types of stress (27). p21 is under the transcriptional control of p53 gene and is a universal inhibitor of cyclin kinases (25). Cyclin D1, a well-known nuclear protein, has been implicated in cell cycle control (28). According to our data, emodin attenuated the upregulation of $p 53$ and $p 21$, as well as the down-regulation of cyclin D1 in hypoxia-treated cells, which indicated that emodin increased cell proliferation. This result indicated that emodin protected $\mathrm{H} 9 \mathrm{c} 2$ cells from hypoxia-induced injury. In addition, the important mediators of apoptosis, 


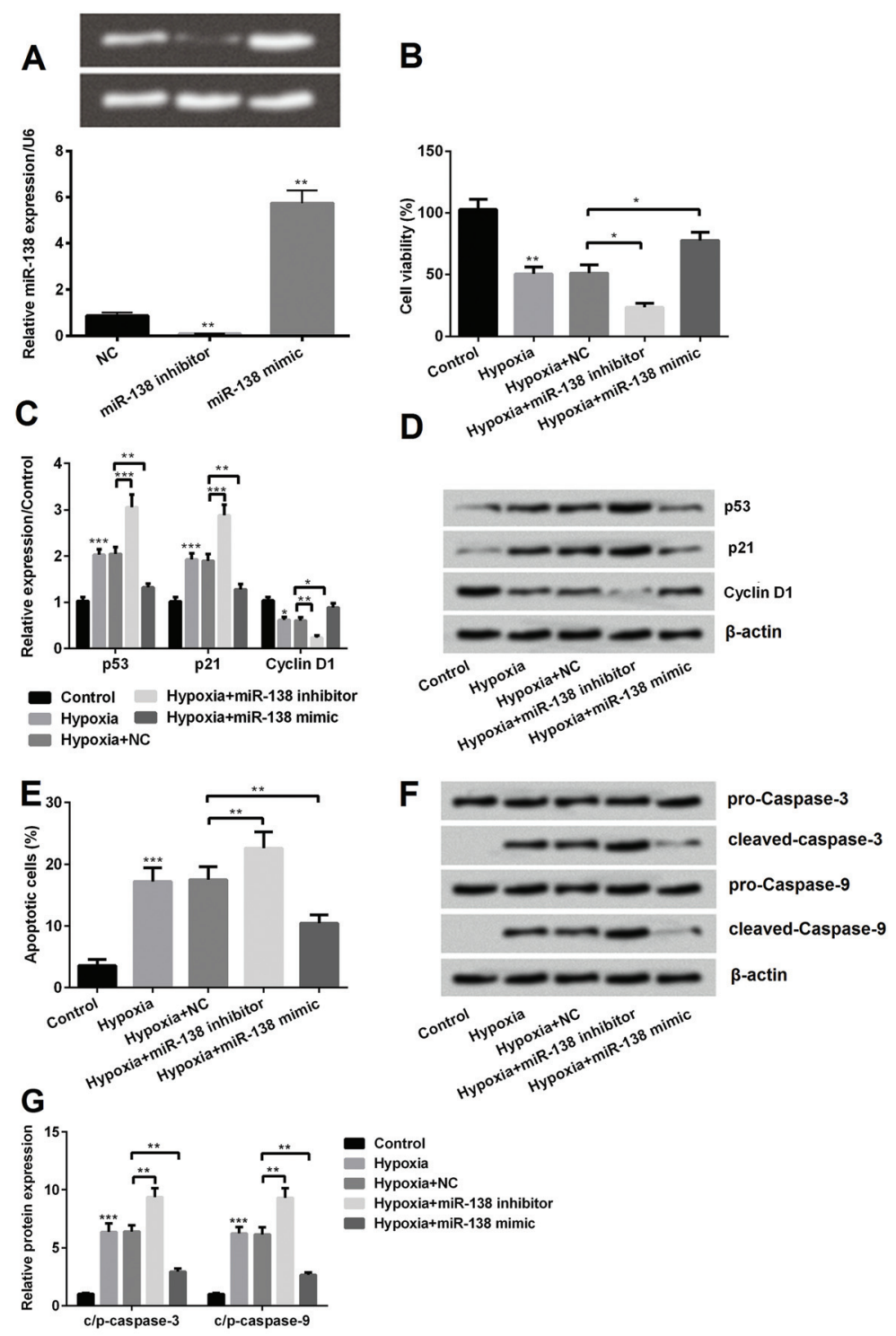

Figure 4. miR-138 overexpression alleviated hypoxiainduced cell injury. A, miR-138 expression was upregulated or down-regulated after transfection assay analyzed by qRT-PCR. $B$, miR-138 overexpression up-regulated in hypoxia-treated cells compared with negative control (NC). $C$ and $D$, The protein expressions of p53 and p21 were up-regulated and cyclin D1 was down-regulated by miR-138 overexpression compared with NC. $E$ and $F$, miR-138 overexpression decreased cell apoptosis and the effects on apoptosis-related proteins were detected by western blot. Data are reported as means \pm SD of triplicates. Each experiment was performed three times. ${ }^{*} \mathrm{P}<0.05,{ }^{* *} \mathrm{P}<0.01,{ }^{* * *} \mathrm{P}<0.001$ compared to control or as indicated (one-way analysis of variance).

caspase- 3 and caspase- 9 , were activated after hypoxia but were down-regulated by emodin treatment.

A recent study demonstrated that emodin protects hyperglycemia-induced injury in PC-12 cells by upregulating miR-9. Meanwhile, these observations were coupled with the down-regulation of p21, p16, Bax, cleaved-caspase- 3 and -9 , and the up-regulation of cyclin D1 and Bcl-2 (29), which was consistent with our study. Furthermore, emodin attenuated LPS- and hypoxia/ reoxygenation-induced barrier dysfunction in intestinal epithelial (8), which confirmed the hypoxia injury-inhibitory effects of emodin. Taken together, emodin revealed cytoprotective effects, which are consistent with previous studies.
Emodin was reported to protect cells by regulation of miRNAs expression including miR-34a (13), miR-126 (12), and miR-30a-5p (30). Simultaneously, MI was also closely related with miRNAs, such as miR-1 (31) and miR-21 (32). Among these miRNAs, the role of miR-138 in cardiocytes was reported in recent studies. These studies showed that up-regulating miR-138 inhibited hypoxia-induced cardiomyocyte apoptosis (15). Another study demonstrated that miR-138 could protect cardiomyocytes from hypoxiainduced apoptosis (33). The protective effect of miR-138 against cerebral ischemia/reperfusion injury in rats was displayed (34). In our study, miR-138 expression was significantly down-regulated by hypoxia and up-regulated by emodin in $\mathrm{H} 9 \mathrm{c} 2$ cells. This result was consistent with 
A

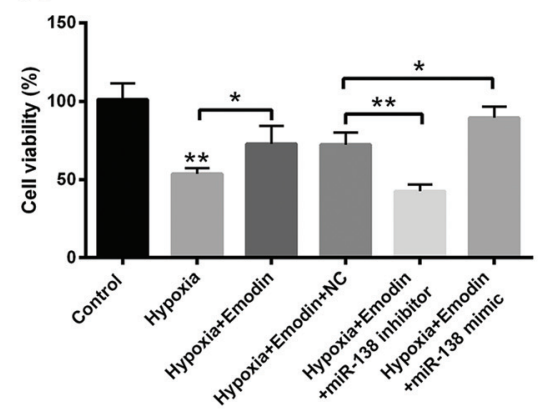

C

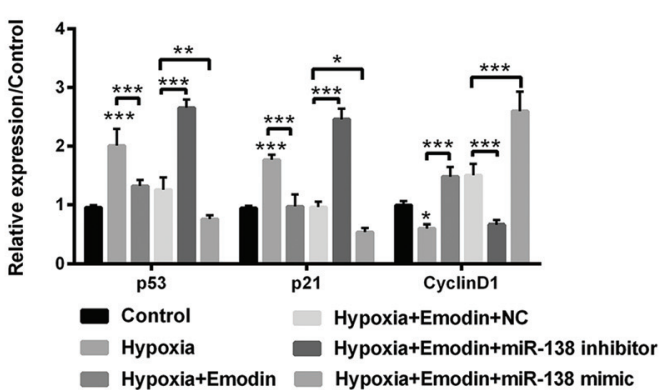

E
B

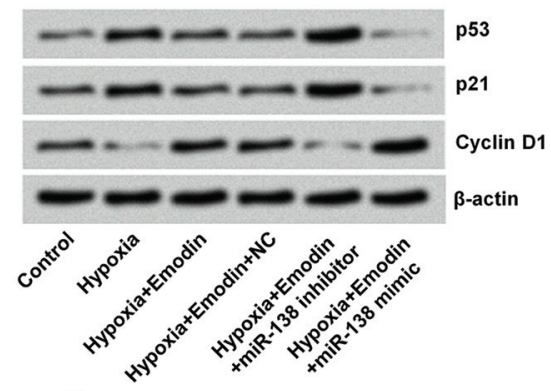

D

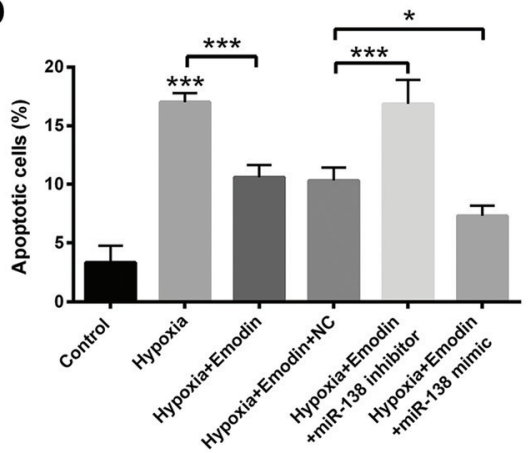

\section{$\mathbf{F}$}

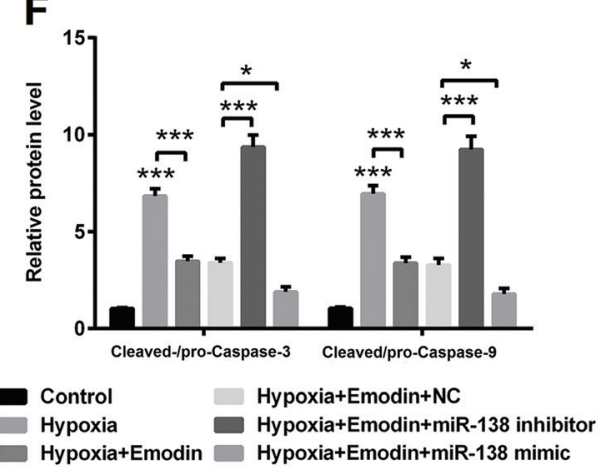

Figure 5. miR-138 modulated the effects of emodin on hypoxia injury in $\mathrm{H} 9 \mathrm{c} 2$ cells. $A$, miR-138 positively regulated viability of emodintreated cells detected by CCK-8 assay. $B$, miR-138 was involved in the effects of emodin on proliferation-related protein expression. $C$, miR-138 negatively regulated apoptosis of emodin-treated cells detected by flow cytometry. $D-F$, miR-138 was involved in the effects of emodin on apoptosis-related protein expression. The expression of protein was measured by western blot. Data are reported as means $\pm S D$ of triplicates. Each experiment was performed three times. NC: negative control. ${ }^{*} P<0.05,{ }^{* *} P<0.01,{ }^{* * *} P<0.001$ compared to control or as indicated (one-way analysis of variance).

previous studies in which hypoxia reduced the expression of miR-138 $(15,35)$. However, this result is contrary with the result from $\mathrm{He}$ et al. (33) in which hypoxia increased the expression of miR-138. In the research by $\mathrm{He}$ et al. (33), the authors did serum-free starving treatment before the treatment of hypoxia, which induced their cells into G0 phase, while in our experiment, we did not do the starvation treatment, which kept our cells in
$S$ phase. The expression of miRNAs is affected by different cell phases (36).

Furthermore, our results demonstrated protective functions of miR-138, which were consistent with the studies that miR-138 protected against hypoxia-induced cell injury $(15,33)$. miR-138 silence impaired the protective effect of emodin against hypoxia-induced injury but miR138 overexpression enhanced the protective effect of 
A
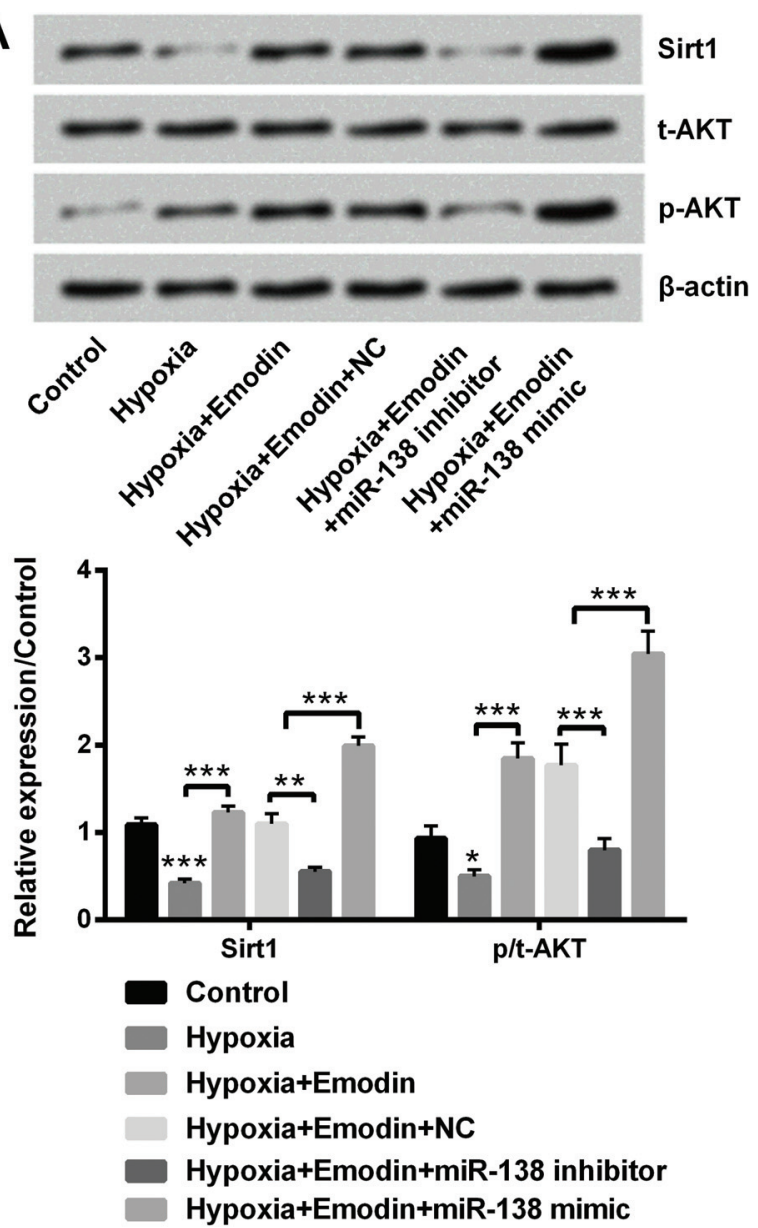

B

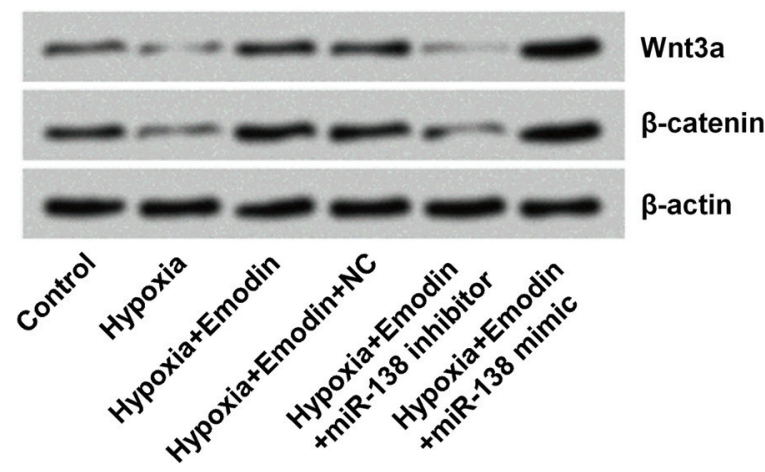

Figure 6. Sirt1/AKT and Wnt/ $\beta$-catenin signaling pathways were activated by emodin. Emodin promoted activations of $(A)$ Sirt $1 / A K T$ pathway and $(B) \mathrm{Wnt} / \beta$-catenin pathway by up-regulating miR-138. The expression of protein was measured by western blot. Data are reported as means $\pm S D$ of triplicates. Each experiment was performed three times. NC: negative control. ${ }^{*} P<0.05$, ${ }^{* *} P<0.01$, ${ }^{* * *} \mathrm{P}<0.001$ compared to control or as indicated (one-way analysis of variance).

emodin, which indicated that emodin showed the protective effects through up-regulation of miR-138. This result was consistent with a previous study that showed that miR-138 might be able to modulate efficacy of emodin in hypoxia-treated $\mathrm{H} 9 \mathrm{c} 2$ cells (11).

Nicotinamide adenosine dinucleotide (NAD)-dependent deacetylase Sirt1 is a type of NAD-dependent deacetylase and has proven to play important roles in multiple biological functions, particularly anti-apoptosis (37). Sirt1 overexpression could promote cell viability and decrease apoptosis of hypoxia-treated osteoblast cells. In addition, Sirt1 activated anti-apoptotic effects by decreasing the activity of caspase-3, -9 , and subsequent pathways (38). In our study, under emodin treatment, Sirt1/AKT signaling pathway was activated by miR-138 overexpression in hypoxia-treated cells and the activation of Sirt1/AKT pathway might partly explain the anti-apoptotic property of emodin.
Wnt/ $\beta$-catenin pathway was also activated by emodin in this study. Wnt/ $\beta$-catenin pathway was shown to promote hepatocyte survival in vivo under hypoxic conditions (39), which was consistent with our study.

MLK3 was reported to co-work with miR-138 in hypoxiainduced injury cells (33). In our study, we found that MLK3 was negatively modulated by miR-138 expression. In addition, MLK3 was a target of miR-138, and miR-138 could inhibit the expression of MLK3 and further influence the synthesis of MLK3 protein, and then affect some biological functions in cells. With miR-138 knockdown, the effects on MLK3 were alleviated, causing MLK3 overexpression in $\mathrm{H} 9 \mathrm{c} 2$ cells, which inactivated the Sirt1/AKT and $W n t / \beta$-catenin pathways. This cascade reaction might be an explanation about how miR-138 affected cell functions.

The results from the present study demonstrated that emodin might have a protective function in hypoxia-induced 

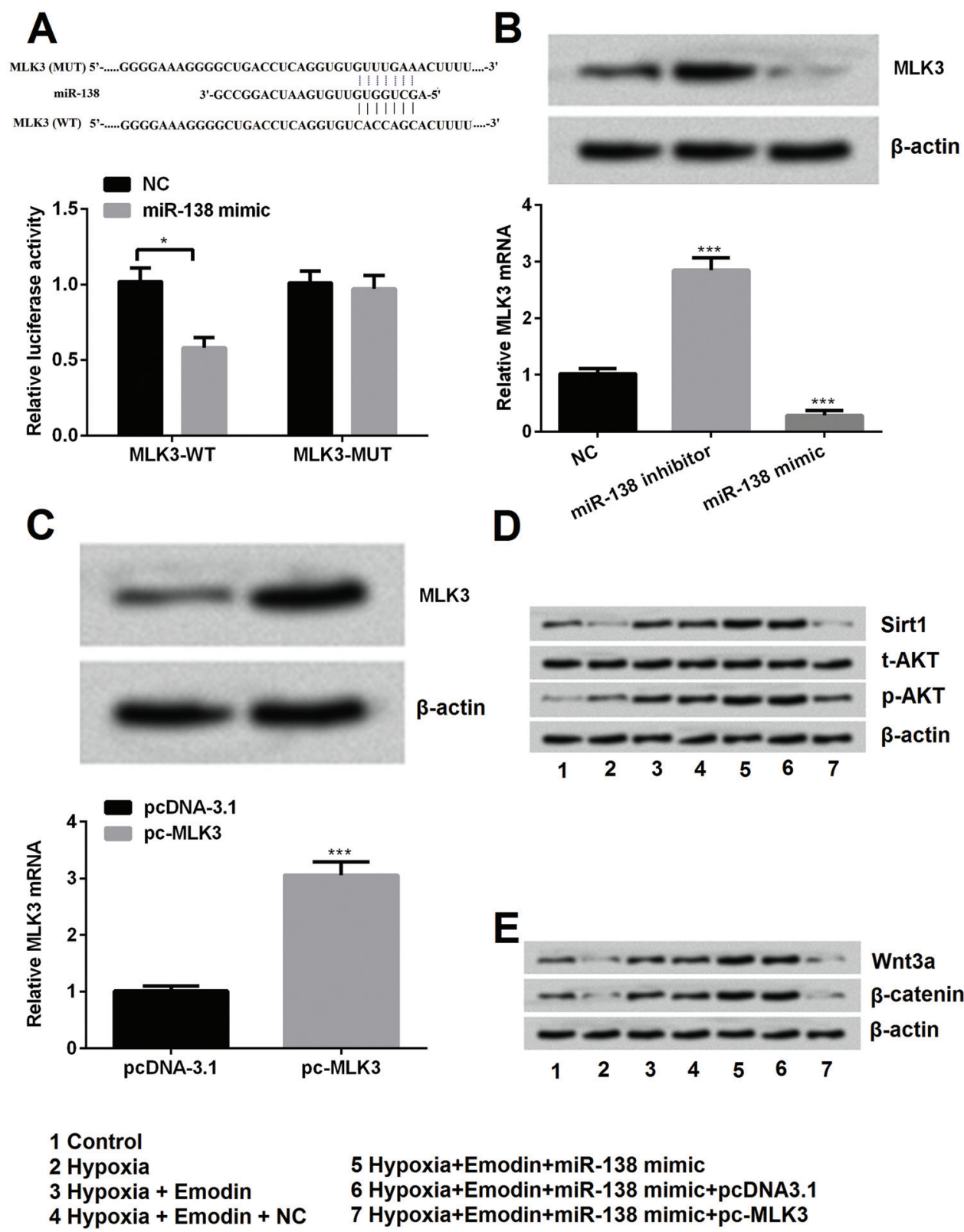

Figure 7. Emodin alleviated hypoxia-induced cell injury via downregulation of MLK3. $A$, Dual luciferase activity assay was performed to confirm miR-138 target MLK3. B, miR-138 negatively regulated expression of MLK3. C, Overexpression of MLK3 was accessed by transfection with pc-MLK3. Under emodin treatment, $(D)$ Sirt1/AKT pathway and $(E) \mathrm{Wnt} / \beta$-catenin pathway were inactivated by cotransfection with miR-138 mimic and pc-MLK3 compared with negative control (NC). The expression of protein was measured by western blot. Data are reported as means $\pm S D$ of triplicates. Each experiment was performed three times. ${ }^{*} P<0.05$, ${ }^{* * *} P<0.001$, Student's $t$-test and one-way analysis of variance.

injury in H9c2 cells. Emodin promoted cell viability and inhibited cell apoptosis induced by hypoxia possibly by upregulating miR-138, during which the activations of Sirt1/
AKT and Wnt/ $\beta$-catenin pathways were essential. Emodin and miR-138 intervention may potentially aid in the treatment of ischemic heart disease. 


\section{References}

1. Sahoo S, Losordo DW. Exosomes and cardiac repair after myocardial infarction. Circ Res 2014; 114: 333-344, doi: 10.1161/CIRCRESAHA.114.300639.

2. Shah AS, McAllister DA, Mills R, Lee KK, Churchhouse AM, Fleming KM, et al. Sensitive troponin assay and the classification of myocardial infarction. The Am J Med 2015; 128 : 493-501, doi: 10.1016/j.amjmed.2014.10.056.

3. Vdovin N, Günther SPW, de Waha S, Seizer P, Brunner S, Schlensak $C$, et al. Early risk stratification in patients with cardiogenic shock complicating acute myocardial infarction treated with extracorporeal life support and primary percutaneous coronary intervention. JACC: Cardiovascular Interventions 2017; 10: 2469-2471, doi: 10.1016/j.jcin.2017.08. 055.

4. Haque E, Kamil M, Irfan S, Sheikh S, Hasan A, Nazir A, et al. Blocking mutation independent p53 aggregation by emodin modulates autophagic cell death pathway in lung cancer. Int J Biochem Cell Biol 2018; 96: 90-95, doi: 10.1016/ j.biocel.2018.01.014.

5. Dai JP, Wang QW, Su Y, Gu LM, Zhao Y, Chen XX, et al. Emodin inhibition of influenza a virus replication and influenza viral pneumonia via the Nrf2, TLR4, p38/JNK and NF-kappaB pathways. Molecules 2017; 22: 1754, doi: 10.3390/molecules 22101754

6. Duan F, Xin G, Niu H, Hunag W. Chlorinated emodin as a natural antibacterial agent against drug-resistant bacteria through dual influence on bacterial cell membranes and DNA. Sci Rep 2017; 7: 12721, doi: 10.1038/s41598-017-12905-3.

7. Tian SL, Yang $\mathrm{Y}$, Liu $\mathrm{XL}, \mathrm{Xu} \mathrm{QB}$. Emodin attenuates bleomycin-induced pulmonary fibrosis via anti-inflammatory and anti-oxidative activities in rats. Med Sci Monit 2018; 24: 1-10, doi: 10.12659/MSM.905496.

8. Lei Q, Qiang F, Chao D, Di W, Guoqian Z, Bo Y, et al. Amelioration of hypoxia and LPS-induced intestinal epithelial barrier dysfunction by emodin through the suppression of the NF-kappaB and HIF-1alpha signaling pathways. Int $J$ Mol Med 2014; 34: 1629-1639, doi: 10.3892/jmm.2014. 1965.

9. Wu J, Ke X, Wang W, Zhang H, Ma N, Fu W, et al. Aloeemodin suppresses hypoxia-induced retinal angiogenesis via inhibition of HIF-1 $\alpha$ /VEGF pathway. Int J Biol Sci 2016; 12: 1363-1371, doi: 10.7150/ijbs.16334.

10. He L, Hannon GJ. MicroRNAs: small RNAs with a big role in gene regulation. Nat Rev Genet 2004; 5: 522-531, doi: $10.1038 / n r g 1379$.

11. Rukov JL, Shomron N. MicroRNA pharmacogenomics: Post-transcriptional regulation of drug response. Trends Mol Med 2011; 17: 412-423, doi: 10.1016/j.molmed.2011. 04.003.

12. Hua JY, He YZ, Xu Y, Jiang $X H$, Ye W, Pan ZM. Emodin prevents intima thickness via Wnt4/Dvl-1/beta-catenin signaling pathway mediated by miR-126 in balloon-injured carotid artery rats. Exp Mol Med 2015; 47: e170, doi: 10.1038/emm.2015.36

13. Guo J, Li W, Shi H, Xie X, Li L, Tang H, et al. Synergistic effects of curcumin with emodin against the proliferation and invasion of breast cancer cells through upregulation of miR34a. Mol Cell Biochem 2013; 382: 103-111, doi: 10.1007/ s11010-013-1723-6.
14. Song $\mathrm{K}$, Lv T, Chen $\mathrm{Y}$, Diao $\mathrm{Y}$, Yao Q, Wang $\mathrm{Y}$. Emodin inhibits TGF-beta2 by activating the FOXD3/miR199a axis in ovarian cancer cells in vitro. Oncol Rep 2018; 39: 20632070, doi: 10.3892/or.2018.6301.

15. Xiong $\mathrm{H}$, Luo T, He W, Xi D, Lu H, Li M, et al. Up-regulation of miR-138 inhibits hypoxia-induced cardiomyocyte apoptosis via down-regulating lipocalin-2 expression. Exp Biol Med 2016; 241: 25-30, doi: 10.1177/1535370215591831.

16. Salloum FN, Yin C, Kukreja RC. Role of microRNAs in cardiac preconditioning. J Cardiovasc Pharmacoly 2010; 56: 581-588, doi: 10.1097/FJC.0b013e3181f581ba.

17. Li H, Wang R. Blocking SIRT1 inhibits cell proliferation and promotes aging through the PI3K/AKT pathway. Life Sci 2017; 190: 84-90, doi: 10.1016/j.Ifs.2017.09.037.

18. Wang G, Wang JJ, To TS, Zhao HF, Wang J. Role of SIRT1mediated mitochondrial and Akt pathways in glioblastoma cell death induced by Cotinus coggygria flavonoid nanoliposomes. Int J Nanomedicine 2015; 10: 5005-5023, doi: 10.2147/IJN. S82282.

19. Ling S, Li J, Shan Q, Dai H, Lu D, Wen X, et al. USP22 mediates the multidrug resistance of hepatocellular carcinoma via the SIRT1/AKT/MRP1 signaling pathway. Mol Oncol 2017; 11: 682-695, doi: 10.1002/1878-0261.12067.

20. Zhu J, Shi H, Liu H, Wang X, Li F. Long non-coding RNA TUG1 promotes cervical cancer progression by regulating the miR-138-5p-SIRT1 axis. Oncotarget 2017; 8: 6525365264, doi: 10.18632/oncotarget.18224.

21. Livak KJ, Schmittgen TD. Analysis of relative gene expression data using real-time quantitative PCR and the 2(-Delta Delta C(T)) Method. Methods 2001; 25: 402-408, doi: 10.1006/meth.2001.1262.

22. Samuel SM, Thirunavukkarasu M, Penumathsa SV, Paul D, Maulik N. Akt/FOXO3a/SIRT1-mediated cardioprotection by $n$-tyrosol against ischemic stress in rat in vivo model of myocardial infarction: switching gears toward survival and longevity. J Agric Food Chem 2008; 56: 9692-9698, doi: 10.1021/jf802050h

23. Blankesteijn WM, van Gijn ME, Essers-Janssen YP, Daemen MJ, Smits JF. Beta-catenin, an inducer of uncontrolled cell proliferation and migration in malignancies, is localized in the cytoplasm of vascular endothelium during neovascularization after myocardial infarction. Am J Pathol 2000; 157: 877-883, doi: 10.1016/S0002-9440(10)64601-9.

24. Monisha BA, Kumar N, Tiku AB. Emodin and Its role in chronic diseases. Adv Expl Med Biol 2016; 928: 47-73, doi: 10.1007/978-3-319-41334-1.

25. Xiong $\mathrm{Y}$, Hannon GJ, Zhang $\mathrm{H}$, Casso D, Kobayashi R, Beach D. p21 is a universal inhibitor of cyclin kinases. Nature 1993; 366: 701-704, doi: 10.1038/366701a0.

26. Bukholm IK, Nesland JM. Protein expression of p53, p21 (WAF1/CIP1), bcl-2, Bax, cyclin D1 and pRb in human colon carcinomas. Virchows 2000; 436: 224-228, doi: 10.1007/ s004280050034.

27. Haupt $\mathrm{Y}$, Maya R, Kazaz A, Oren M. Mdm2 promotes the rapid degradation of p53. Nature 1997; 387: 296-299, doi: 10.1038/387296a0

28. Baldin V, Lukas J, Marcote MJ, Pagano M, Draetta G. Cyclin $\mathrm{D} 1$ is a nuclear protein required for cell cycle progression in G1. Genes Dev 1993; 7: 812-821, doi: 10.1101/gad.7.5.812. 
29. Fan L, Zhang H, Li X, Yang G, Ru J, Liu T. Emodin protects hyperglycemia-induced injury in PC-12cells by up-regulation of miR-9. Mol Cell Endocrinol 2018; 474: 194-200, doi: 10.1016/j.mce.2018.03.009.

30. Xiang H, Tao X, Xia S, Qu J, Song H, Liu J, et al. Emodin Alleviates sodium taurocholate-induced pancreatic acinar cell injury via MicroRNA-30a-5p-mediated inhibition of hightemperature requirement $\mathrm{A} /$ transforming growth factor Beta 1 inflammatory signaling. Front Immunol 2017; 8: 1488, doi: 10.3389/fimmu.2017.01488.

31. Ai J, Zhang R, Li Y, Pu J, Lu Y, Jiao J, et al. Circulating microRNA-1 as a potential novel biomarker for acute myocardial infarction. Biochem Biophys Res Commun 2010; 391: 73-77, doi: 10.1016/j.bbrc.2009.11.005.

32. Roy S, Khanna S, Hussain SR, Biswas S, Azad A, Rink C, et al. MicroRNA expression in response to murine myocardial infarction: miR-21 regulates fibroblast metalloprotease-2 via phosphatase and tensin homologue. Cardiovasc Res 2009; 82: 21-29, doi: 10.1093/cvr/cvp015.

33. He S, Liu P, Jian Z, Li J, Zhu Y, Feng Z, et al. miR-138 protects cardiomyocytes from hypoxia-induced apoptosis via MLK3/JNK/c-jun pathway. Biochem Biophys Res Commun 2013; 441: 763-769, doi: 10.1016/j.bbrc.2013. 10.151.
34. Tang XJ, Yang MH, Cao G, Lu JT, Luo J, Dai LJ, et al. Protective effect of microRNA-138 against cerebral ische$\mathrm{mia} /$ reperfusion injury in rats. Exp Ther Med 2016; 11: 10451050, doi: 10.3892/etm.2016.3021.

35. Li S, Ran Y, Zhang D, Chen J, Li S, Zhu D. MicroRNA-138 plays a role in hypoxic pulmonary vascular remodelling by targeting Mst1. Biochem J 2013; 452: 281-291, doi: 10.1042/BJ20120680.

36. Carleton M, Cleary MA, Linsley PS. MicroRNAs and cell cycle regulation. Cell Cycle 2007; 6: 2127-2132, doi: 10.4161/ cc.6.17.4641.

37. Wang P, Xu TY, Guan YF, Tian WW, Viollet B, Rui YC, et al. Nicotinamide phosphoribosyltransferase protects against ischemic stroke through SIRT1-dependent adenosine monophosphate-activated kinase pathway. Ann Neurol 2011; 69: 360-374, doi: 10.1002/ana.22236.

38. Zhou L, Wang SI, Moon YJ, Kim KM, Lee KB, Park BH, et al. Overexpression of SIRT1 prevents hypoxiainduced apoptosis in osteoblast cells. Mol Med Rep 2017; 16: 2969-2975, doi: $10.3892 / \mathrm{mmr} .2017 .6917$.

39. Lehwald N, Tao GZ, Jang KY, Sorkin M, Knoefel WT, Sylvester KG. Wnt-beta-catenin signaling protects against hepatic ischemia and reperfusion injury in mice. Gastroenterology 2011; 141: 707-718, 718. e1-5, doi: 10.1053/ j.gastro.2011.04.051. 